\title{
Benefits and applications of laser-induced sparks in real scale model measurements
}

Gómez-Bolaños, Javier; Delikaris-Manias, Symeon; Pulkki, Ville Topias; Eskelinen, Joona; Hæggström, Edward; Jeong, Cheol-Ho

Published in:

J A S A Express Letters

Link to article, DOI:

$10.1121 / 1.4929623$

Publication date:

2015

Document Version

Publisher's PDF, also known as Version of record

Link back to DTU Orbit

Citation (APA):

Gómez-Bolaños, J., Delikaris-Manias, S., Pulkki, V. T., Eskelinen, J., Hæggström, E., \& Jeong, C-H. (2015). Benefits and applications of laser-induced sparks in real scale model measurements. J A S A Express Letters, 138(3), EL175-EL180. https://doi.org/10.1121/1.4929623

\section{General rights}

Copyright and moral rights for the publications made accessible in the public portal are retained by the authors and/or other copyright owners and it is a condition of accessing publications that users recognise and abide by the legal requirements associated with these rights.

- Users may download and print one copy of any publication from the public portal for the purpose of private study or research.

- You may not further distribute the material or use it for any profit-making activity or commercial gain

- You may freely distribute the URL identifying the publication in the public portal 


\title{
Benefits and applications of laser-induced sparks in real scale model measurements
}

\author{
Javier Gómez-Bolaños, ${ }^{\text {a) }}$ Symeon Delikaris-Manias, and Ville Pulkki \\ Department of Signal Processing and Acoustics, Aalto University, P.O. Box 13000, \\ Espoo FI-00076, Finland \\ javier.gomez.bolanos@aalto.fi,symeon.delikaris-manias@aalto.fi,ville.pulkki@aalto.fi \\ Joona Eskelinen and Edward Hæggström \\ Department of Physics, University of Helsinki, P.O. Box 64, Helsinki FI-00014, Finland \\ joona.eskelinen@helsinki.fi,edward.haeggstrom@helsinki.fi \\ Cheol-Ho Jeong \\ Department of Electrical Engineering, Technical University of Denmark, Ørsteds Plads, \\ Building 352, Lyngby, Denmark \\ chj@elektro.dtu.dk
}

\begin{abstract}
The characteristics of using a laser-induced spark as a monopole source in scale model measurements were assessed by comparison with an electric spark and a miniature spherical loudspeaker. Room impulse responses of first order directivity sources were synthesized off-line using six spatially distributed sparks. The source steering direction was scanned across the horizontal and vertical plane to assess the origin of early reflections. The results confirm that the characteristics of the laser-induced spark outperform those of typical sources. Its monopole characteristics enable the authors to synthesize room responses of directional sources, e.g., to obtain directional information about reflections inside scale models.
\end{abstract}

(C) 2015 Acoustical Society of America

[NX]

Date Received: May 22, 2015 Date Accepted: August 12, 2015

\section{Introduction}

Scale models permit studying the acoustic behavior of large acoustic systems, especially at low frequencies. Although physical scale models are largely replaced by simulation software, they are still used in sound diffusion studies, ${ }^{1}$ auralization, ${ }^{2}$ and evaluation of computer simulations. ${ }^{3}$ Unfortunately, typical scale model measurements provide a limited insight about the model. In typical scale model measurements, a propagation path from an omnidirectional source to an omnidirectional receiver is usually assumed. Therefore, the measurement lacks directional information about the origin of the reflections. This information can be obtained, for instance, by steering a directional source and analyzing the measured responses for different steering directions.

Directional sources can be synthesized by combining several monopole sources by means of beamforming techniques. ${ }^{4}$ An ideal monopole source should be omnidirectional and point-like to avoid undesired reflections from the source itself. Miniature spherical loudspeakers and electric sparks are commonly used in scale model measurements to minimize the effect of the source volume. Spherical loudspeakers feature an omnidirectional radiation pattern at low frequencies. However, they emit a multidirectional radiation pattern at mid and high frequencies due to the separation of the different source elements. The effect of the multidirectional radiation decreases the accuracy of the measured responses. ${ }^{5}$ Moreover, loudspeakers feature a finite volume that may shadow or reflect sound. For these reasons, miniature loudspeakers cannot be regarded as a true monopole source. On the other hand, electric sparks with a narrow interelectrode gap are tiny and omnidirectional, ${ }^{6}$ but the electrode stand occupies a finite volume that may affect the measured response of the scale model. Therefore, nonintrusive methods would be optimal to avoid the effect of the source.

The laser-induced air breakdown, LIB, is generated by focusing a high energy pulsed laser into one point in air producing a spark. Such a LIB spark can be nonintrusively placed inside a model, e.g., by focusing the laser beam through a glass window. ${ }^{7}$ The LIB produces a high level impulse that is omnidirectional, repeatable,

\footnotetext{
${ }^{\text {a) }}$ Author to whom correspondence should be addressed.
} 
broadband, and provides a response suitable for acoustic measurements of scale models. ${ }^{7}$ However, its performance has not yet been compared to typical sources. The monopole characteristics of LIB suggest that it can be optimal for synthesizing the response of directional sources inside the scale model by combining the measured impulse responses generated by several spatially close sparks. ${ }^{8}$ These directional responses can then be analyzed to obtain information about the origin of the reflections or be used for auralization.

In this work, the room impulse responses measured using LIB, a spherical loudspeaker, and an electric spark are compared to assess the suitability of LIB in a real scale model. In addition, six spatially separated LIBs are used to produce off-line first order source directivity. This virtual directional source is then scanned in the horizontal and vertical planes to determine the origin and path of early reflections captured at a single receiver position.

\section{Apparatus and methods}

\subsection{Measurement setup}

A LIB source, an electric spark, and a miniature dodecahedron loudspeaker were used to measure a 1:10 scale model of the National Concert Hall in Dublin (located at Technical University of Denmark, DTU). The LIB source consists of a pulsed laser (CRF400, $400 \mathrm{~mJ}, 1064 \mathrm{~nm}, 8 \mathrm{~ns}$, Quantum, France) focused by a $50 \mathrm{~mm}$ focusing lens (LA1131, Thorlabs, Newton, NJ). The laser setup was placed under the stage of the scale model. The laser beam was pointed upwards using a mirror (NB1-K13, Thorlabs, Newton, NJ) and focused $40 \mathrm{~mm}$ above the stage through a glass window $(2 \mathrm{~mm} \mathrm{H}$, $120 \mathrm{~mm} \mathrm{~W}, 120 \mathrm{~mm} \mathrm{~L}$ ). The laser head, mirror, and lens were installed on a custombuilt platform that allowed shifting the breakdown position along the $X, Y$, and $Z$ axis [see Fig. 1(a)].

The electric spark $(750 \mathrm{~V} / 28 \mathrm{~kW}$, custom-made at DTU) consisted of three tungsten electrodes mounted on a cylindric stand and separated by $1 \mathrm{~mm}$. The electrode stand was beneath the stage and the electrodes were brought into the model through an acrylic plate $(2 \mathrm{~mm} \mathrm{H}, 120 \mathrm{~mm} \mathrm{~W}, 120 \mathrm{~mm} \mathrm{~L})$ [see Fig. 1(a)]. The miniature dodecahedron loudspeaker (custom-made at DTU) consists of 12 piezoelectric loudspeakers $(\varnothing=15 \mathrm{~mm})$. The loudspeaker is hollow inside and its diameter is $60 \mathrm{~mm}$. The loudspeaker stand was placed in a similar manner as the stand of the electric spark. The loudspeaker amplifier was placed outside the scale model. The source position was $40 \mathrm{~mm}$ above the stage and $80 \mathrm{~mm}$ from the middle axis of the stage.

The receiver was a $1 / 8$ in. pressure microphone (type 4138 , Brüel \& Kjær, Denmark) connected to a signal conditioner (Nexus, Brüel \& Kjær, Denmark). The receiver position was located approximately $40 \mathrm{~mm}$ below the horizontal plane of the source and centered in the scale model. The LIB and electric spark measurements were digitized at $4 \mathrm{MHz}$ sampling rate using a data acquisition device (NI4952, National Instruments, Austin, TX) connected to a computer running Labview (National

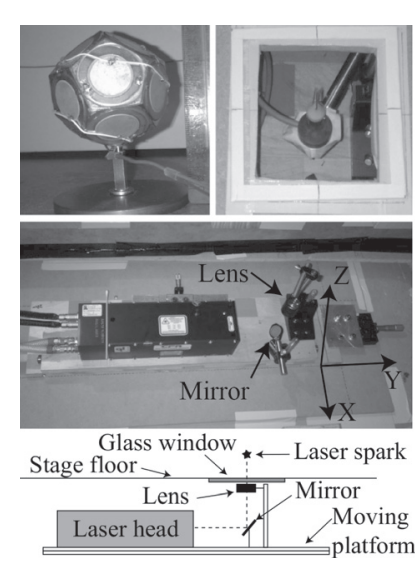

(a)

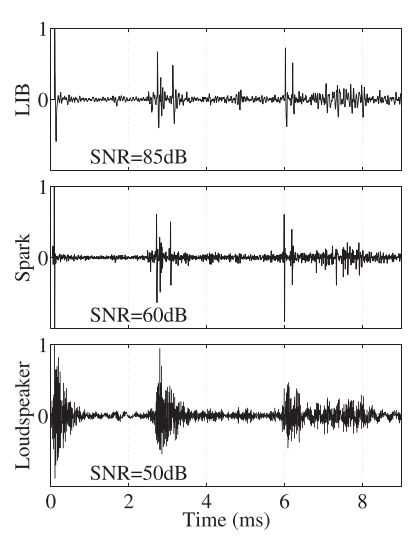

(b)

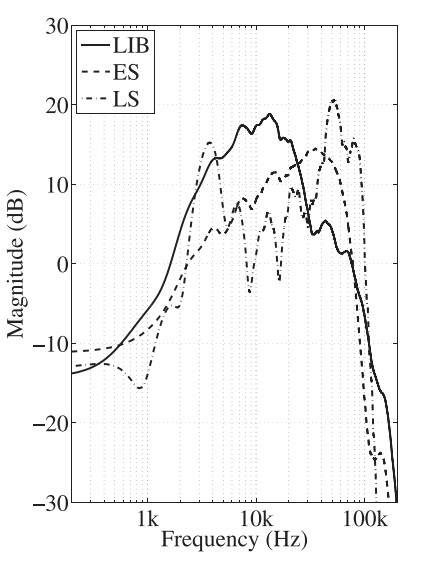

(c)

Fig. 1. (a) Spherical loudspeaker (top-left), electric spark (top-right), and laser spark (center) used in the measurements. Sketch of the laser device and optics below the stage floor (bottom). (b) First $9 \mathrm{~ms}$ of the normalized impulse responses measured at the same receiver position inside the model for LIB (top), electric spark (center), and spherical loudspeaker (bottom). (c) Normalized magnitude responses of the DS (first $1 \mathrm{~ms}$ ) obtained with LIB (solid line), electric spark (dashed line), and spherical loudspeaker (dashed-dotted line) (1/3 octave smoothing). 
Instruments, Austin, TX). The measurements using LIB and the electric spark were 20 times averaged to avoid the effects of external factors, e.g., dust, in the stability of the pulse waveform and spark position. ${ }^{7,8}$ The standard deviation of the peak value over 20 repetitions was $0.2 \mathrm{~dB}$. Additionally, the averaging process introduces an improvement on the signal-to-noise ratio (SNR) of $13 \mathrm{~dB}$. The loudspeaker measurements were done using a $10 \mathrm{~s}$ long sweep and digitized at $200 \mathrm{kHz}$ sampling rate using a different data acquisition device (NI4642, National Instruments, Austin, TX). The duration of the sweep was selected to be the same than the duration of the 20 LIB measurements.

\subsection{Source comparison}

The room impulse responses obtained with the three sources at the same receiver position were compared. The comparison was performed in terms of discrimination between reflections, SNR, magnitude response of the direct sound (DS), and effect of source directivity. The reflection discrimination was done by analyzing the first $9 \mathrm{~ms}$ of the normalized room responses. The SNR of each room response measurement was determined as the ratio between the peak level of the DS and the noise floor at the end of the room response. The magnitude response of the DS was calculated as the fast Fourier transform of the first $1 \mathrm{~ms}$ of the room responses. The effect of source directivity was assessed by analyzing the effect of deconvolution between the room responses and free-field response. An estimate of the free-field response was obtained by windowing the DS. The use of deconvolution to remove the effect of the source response from the room responses is accurate only if there is sufficient SNR and the source is omnidirectional within the deconvolution bandwidth. For non-omnidirectional sources, the room response after deconvolution shows smeared reflections. The deconvolution was performed by using the band limited inverse of the DS between $500 \mathrm{~Hz}$ and $100 \mathrm{kHz}$ to ensure sufficient SNR for all sources.

\subsection{Synthesis of source directivity}

To obtain the room impulse response of a directional source, six measurements with LIB were performed sequentially at the same receiver position. The LIB positions form a 6-point array with two $4 \mathrm{~mm}$ separated points per axis and with spatial aliasing frequency at $42.875 \mathrm{kHz}$. This frequency is calculated as the ratio between the speed of sound $(343 \mathrm{~m} / \mathrm{s})$ and twice the distance between the most separated sparks $(4 \mathrm{~mm})$. The separation between LIB points is a compromise between spatial aliasing and accuracy in the positioning of the custom-built platform.

The disposition of the LIB points resembles a three-dimensional (3D) intensity probe. For this reason, the method chosen to synthesize the source directivity is based on combining off-line figure-of-eight (dipole) and omnidirectional (monopole) signals to produce first order cardioid directivity. ${ }^{9}$ The omnidirectional signal for a source at the center of the array was generated by taking the average of all points. Three dipoles, one per each axis of the Cartesian coordinates $X Y Z$, were generated by subtracting the two LIB signals produced on the same axis. ${ }^{9}$ The positive lobe of the dipoles was aligned with the positive axis illustrated in Fig. 1(a) (center). The frequency response of the dipole signals were equalized to match the response of the omnidirectional source using the filter

$$
H(f)=S_{\mathrm{W}}(f) S_{\mathrm{ref}}(f),
$$

where $S_{\mathrm{W}}(f)$ is the frequency response of the omnidirectional signal and $S_{\text {ref }}(f)$ is the inverse frequency response of a reference dipole between $500 \mathrm{~Hz}$ and $42 \mathrm{kHz}$. The reference dipole was created by subtracting a $12 \mu$ s (approx. $4 \mathrm{~mm}$ for $343 \mathrm{~m} / \mathrm{s}$ ) delayed version of the LIB pulse from the original LIB. Finally, omnidirectional and dipole signals were low-pass filtered with a cutoff frequency at $42 \mathrm{kHz}$ to avoid spatial aliasing effects.

The cardioid source directivity for a specific direction was calculated as

$$
S_{\text {cardioid }}(f, \theta, \phi)=\frac{S_{\mathrm{W}}(f)}{2}+\frac{1}{2}\left[\begin{array}{lll}
S_{\mathrm{X}}(f) & S_{\mathrm{Y}}(f) & S_{\mathrm{Z}}(f)
\end{array}\right]\left[\begin{array}{c}
\cos (\theta) \cos (\phi) \\
\sin (\theta) \cos (\phi) \\
\sin (\theta) \sin (\phi)
\end{array}\right],
$$

where $\theta$ and $\phi$ are the azimuth and elevation angles of the direction toward which the cardioid source points $S_{\mathrm{X}}(f), S_{\mathrm{Y}}(f)$, and $S_{\mathrm{Z}}(f)$ are the frequency responses of the dipoles along the $X, Y$, and $Z$ axes, respectively. The zero-direction $(\phi=\theta=0)$ was along the positive $X$ axis. 
The synthesized source directivity was used to assess the origin of early reflections by finding the maximum level of the received pulses (DS and reflections) in a scan across the horizontal plane (azimuth) and across the vertical plane (elevation). The azimuthal scan was done by setting $\phi=0$ and increasing $\theta$, whereas the elevation scan was done by setting $\theta=0$ and increasing $\phi$. The increments were in steps of $1^{\circ}$ from the positive $X$ axis toward the positive $Y$ axis (azimuth) and toward positive $Z$ axis (elevation) around a complete $360^{\circ}$.

\section{Results}

\subsection{Source comparison}

The first $9 \mathrm{~ms}$ of the normalized room responses obtained with single LIB position (top), electric spark (center), and spherical loudspeaker (bottom) are illustrated in Fig. 1(b). The duration of the LIB and the electric spark pulses enable discrimination of reflections that appear close in time. In contrast, the superposition of the responses of the loudspeaker elements produces a pulse burst. Thus, reflections close in time appear overlapped, as can be seen in the reflections around $3 \mathrm{~ms}$. The LIB signal features the largest SNR between sources, exceeding that of the electric spark by $25 \mathrm{~dB}$ and the loudspeaker by $35 \mathrm{~dB}$.

The normalized magnitude responses of the DS for the three sources are presented in Fig. 1(c). The response of the spherical loudspeaker (dashed-dotted line) presents strong dips caused by the interference between the different elements of the array. The magnitude responses of the LIB and the electric spark are smoother. The electric spark has maximum level around $30 \mathrm{kHz}$ whereas the LIB has maximum level at $15 \mathrm{kHz}$.

The first $9 \mathrm{~ms}$ of the deconvolved room responses are depicted in Fig. 2(a). The omnidirectional radiation of the LIB (top) and the electric spark (center) produces clear peaks at the reflection times, thus being similar to the responses in Fig. 1(b). The room response obtained using the loudspeaker presents smeared reflections due to the directional radiation pattern of this source. This effect is noticeable especially when comparing this response to its equivalent in Fig. 1(b).

\subsection{Synthesis of source directivity}

The first $6.5 \mathrm{~ms}$ of the room response obtained for the omnidirectional, $W$, and dipole sources along the $X, Y$, and $Z$ axes are illustrated in Fig. 2(b). For reflections arriving $3 \mathrm{~ms}$ after the DS, the signal energy is distributed between the $X$ and $Y$ axes, thus suggesting horizontal radiation. In contrast, the reflection arriving $6 \mathrm{~ms}$ after the DS presents signal energy along the $X$ and $\mathrm{Z}$ axes, suggesting vertical radiation.

The result of the azimuthal plane scan is presented in Fig. 2(c), where the first $6.5 \mathrm{~ms}$ of the room response within a dynamic range of $30 \mathrm{~dB}$ is plotted. The same time window of the room response obtained with the monopole source is included at the top of the figure to help identify DS and reflections $(r 1-r 6)$. The cardioid source directivity is seen in the DS. For directions pointing toward the receiver, the signal level

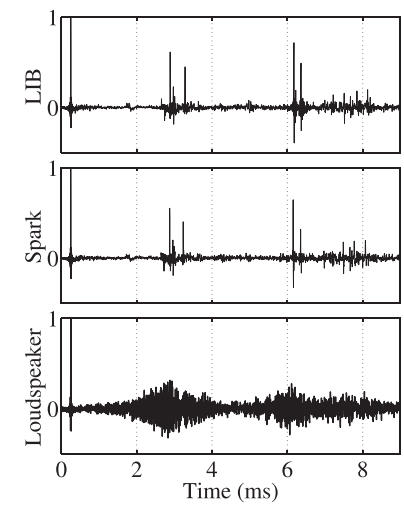

(a)

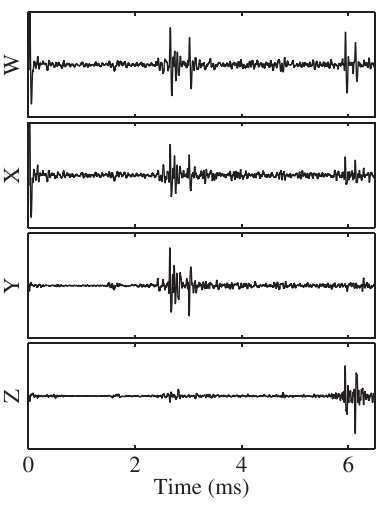

(b)

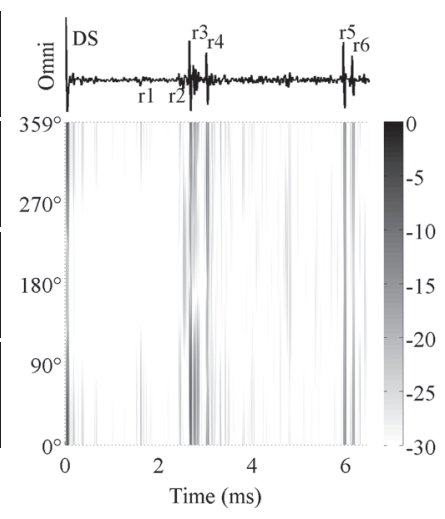

(c)

Fig. 2. (a) First $9 \mathrm{~ms}$ of the normalized impulse responses after deconvolution for LIB (top), electric spark (center), and spherical loudspeaker (bottom). (b) First $6.5 \mathrm{~ms}$ of the impulse responses of the omnidirectional $(W)$ and dipoles directivity along each axis $(X, Y$, and $Z)$. The responses are normalized to the maximum value of $W$. (c) First $6.5 \mathrm{~ms}$ of the impulse responses obtained when scanning across the azimuthal plane. DS and early reflections, $r 1-r 6$, are presented. The cardioid source responses are presented in decibels. 
is maximum, while it decreases when the steering direction changes toward the opposite direction.

Visualizations of the behavior of the DS and the reflections within the first $6.5 \mathrm{~ms}$ for the azimuthal scan are given in Mm. 1 and for the vertical scan in Mm. 2 . These visualizations show the impulse responses obtained when the cardioid source points to distinct scan directions. The amplitude of the DS and reflections vary depending on the source steering direction.

Mm. 1. First $6.5 \mathrm{~ms}$ of the impulse responses when scanning in $1^{\circ}$ steps across the horizontal plane. This file is type "mov" (6.6 MB) [URL: http://dx.doi.org/10.1121/1.4929623.1].

Mm. 2. First $6.5 \mathrm{~ms}$ of the impulse responses when scanning in $1^{\circ}$ steps across the vertical plane. This file is type "mov" (6.6 MB) [URL: http://dx.doi.org/10.1121/1.4929623.2].

The direction of DS and reflections $(r 1-r 6)$ obtained by analyzing the azimuthal and vertical scans are given in Fig. 3(a) as azimuth and elevation. The magnitude of the vectors represents the energy level of the reflections. To identify the reflective surfaces, the results in Fig. 3(a) are overlaid on a picture of the scale model [see Fig. 3(b)].

\section{Discussion}

The spherical loudspeaker features no clear impulse due to the different times of arrival of the signals from the individual elements. This makes reflections close in time hard to discern [see Fig. 1(b)]. The directivity of the spherical loudspeaker affects the deconvolved response producing smeared reflections [see Fig. 2(a)]. The room responses obtained with LIB show similarities to those obtained with the electric spark. Both sources produce a short impulse and clearly identifiable reflections. The LIB and the electric spark are omnidirectional as evidenced by their similar deconvolved responses [see Fig. 2(a)]. The normalized magnitude responses show that the sources feature similar usable bandwidth, that of the LIB being slightly wider than the other sources. If SNR is taken into account, the magnitude response of the electric spark and the loudspeaker should be reduced by 25 and $35 \mathrm{~dB}$, respectively. Therefore, LIB features the widest usable bandwidth. The signal level produced by the electric spark can be increased, although this requires increasing electrode separation which reduces the omnidirectional characteristics of this source. ${ }^{6}$ In addition, since LIB was produced non-intrusively, its position can be moved inside the model without affecting it. To move the electric spark and loudspeaker inside the model, the moving platform needs to be installed inside the model which may affect the response of the scale model.

The monopole and massless features of LIB permits to combine spatially distributed monopole sources with sufficient accuracy to obtain directional information about the scale model or to obtain room responses for directional sources in a nonintrusive manner. Our experiment uses a simple approach by virtually producing cardioid directivity at the source position to scan across the horizontal and vertical planes to identify the origin of the early reflections. When comparing the results in Fig. 3(a) to a picture of the scale model, Fig. 3(b), it can be seen that $r 1$ is produced by the right side stall, $r 2$ is produced by the reflection against the stage floor and the choir balcony [not seen in left side of Fig. 3(b)], $r 3$ is produced by the sound reflecting from the stage floor and then by the right wall and right balcony [top part of Fig. 3(b)], $r 4$ is produced by the left wall [bottom part of Fig. 3(b)] and has a larger amplitude than

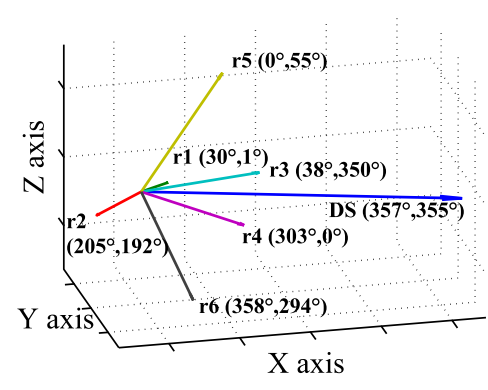

(a)

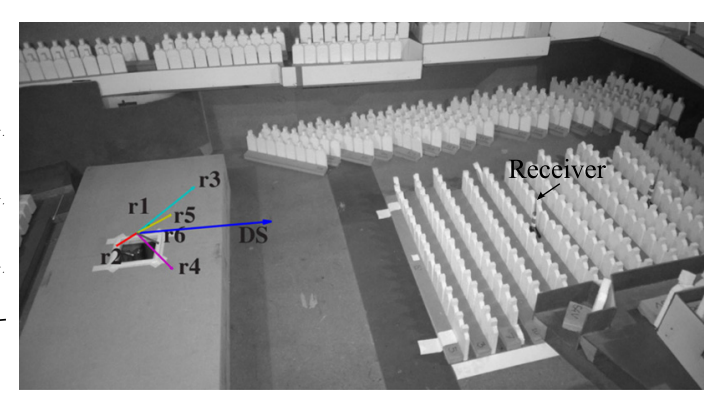

(b)

Fig. 3. (Color online) (a) Estimation of the direction of radiation of the DS and six reflections arriving during the first $6.5 \mathrm{~ms}$. Azimuth and elevation angles are indicated. The length of the vectors is scaled to the energy of the reflection in a $16 \mu$ s window. (b) Estimated directions illustrated in a picture of the model to facilitate recognition of the correspondent reflective surface. 
$r 1$ because the first seven rows of the left side stalls are missing, which makes the sound reflect from a large surface, $r 5$ is produced by the ceiling, and $r 6$ is produced by sound reflecting from the stage floor and ceiling. In addition, the room responses produced with the cardioid sources presented in Mm. 1 and Mm. 2 confirm that the array of LIBs can be used to obtain room responses of directional sources, at least of first order, e.g., for analysis of acoustic parameters obtained with directional sources or for auralization. $^{2}$

Furthermore, by applying the reciprocity principle, the LIB array can be produced at the receiver position and the microphone can be placed at the source position. In this case, the array can be regarded as a 3D intensity probe which permits performing directional analysis at the receiver position. ${ }^{10}$ This opens new possibilities in scale model work, where the directional information can improve the acoustic analysis of the model.

\section{Conclusions}

The room impulse response of a 1:10 scale model obtained using a laser-induced spark was compared to the response obtained with an electric spark and a spherical loudspeaker. The results confirm that LIB can be used in scale model measurements to produce reliable results in a wider bandwidth than with typical sources used in this field. Furthermore, LIB can be produced non-intrusively and can, therefore, be considered to be a true monopole source. In addition, six spatially separated laser sparks were produced and the measured room responses were combined to synthesize first order directional sources. A scan in the horizontal and vertical planes using these directional sources enables one to gather directional information about the origin of the reflections inside the model. This investigation confirms that LIB can be used to carry out directional analysis of the scale model and to obtain impulse responses of first order directional sources.

\section{Acknowledgments}

This work was supported by The Academy of Finland (Project No. 121252) and by the European Research Council under the European Community's Seventh Framework Programme (FP7/2007-2013)/ERC Grant Agreement No. 240453. We express our gratitude to Brüel \& Kjær (Denmark) for providing the microphone and signal conditioner used in the measurements and to Jørgen Rasmussen for his assistance in the measurements.

\section{References and links}

${ }^{1}$ J. K. Ryu and J. Y. Jeon, "Subjective and objective evaluations of a scattered sound field in a scale model opera house," J. Acoust. Soc. Am. 124(3), 1538-1549 (2008).

${ }^{2}$ P. Robinson and N. Xiang, "Design, construction, and evaluation of a 1:8 scale model binaural manikin,” J. Acoust. Soc. Am. 133(3), EL162-EL167 (2013).

${ }^{3}$ P. Luizard, M. Otani, J. Botts, L. Savioja, and B. F. Katz, "Comparison of sound field measurements and predictions in coupled volumes between numerical methods and scale model measurements," Proc. Meet. Acoust. 19(1), 015114 (2013).

${ }^{4}$ L. Beranek and T. Millow, Acoustics: Sound Fields and Transducers (Academic Press, New York, 2012), pp. 142-157.

${ }^{5}$ T. Knüttel, I. B. Witew, and M. Vorländer, "Influence of 'omnidirectional' loudspeaker directivity on measured room impulse responses,” J. Acoust. Soc. Am. 134(5), 3654-3662 (2013).

${ }^{6} \mathrm{C}$. Ayrault, P. Béquine, and S. Baudin, "Characteristics of a spark discharge as an adjustable acoustic source for scale model measurements," in Proceedings of the Acoustics 2012 Nantes Conference, Nantes, France (April 2012).

${ }^{7}$ J. Gómez Bolaños, V. Pulkki, P. Karppinen, and E. Hæggström, “An optoacoustic point source for acoustic scale model measurements,” J. Acoust. Soc. Am. 133(4), EL221-EL227 (2013).

${ }^{8}$ J. Eskelinen, E. Hæggström, S. Delikaris-Manias, J. Gómez Bolaños, and V. Pulkki, "Beamforming with a volumetric array of massless laser spark sources - application in reflection tracking," J. Acoust. Soc. Am. 137(6), EL389-EL395 (2015).

${ }^{9}$ R. A. Rayburn, "First-order directional microphones," in Eargle's The Microphone Book, 3rd ed., edited by R. A. Rayburn (Focal Press, Oxford, 2012), Chap. 5, pp. 81-109.

${ }^{10}$ S. Tervo, J. Pätynen, and T. Lokki, "Acoustic reflection localization from room impulse responses," Acta Acust. Acust. 98(3), 418-440 (2012). 